\title{
Retro-Reflective Fiber Optic Displacement Sensor for Performance Optimization Using Taguchi Method
}

\author{
Supriya S. Patil ${ }^{1}$, A. D. Shaligram² \\ ${ }^{1}$ Department of Electronic Science, Abasaheb Garware College, Pune, Maharashtra, India \\ ${ }^{2}$ Department of Electronic Science, Savitribai Phule Pune University, Pune, Maharashtra, India \\ Email: patilss121@gmail.com
}

How to cite this paper: Patil, S.S. and Shaligram, A.D. (2020) Retro-Reflective Fiber Optic Displacement Sensor for Performance Optimization Using Taguchi Method. Journal of Sensor Technology, 10, 1-13. https://doi.org/10.4236/jst.2020.101001

Received: August 29, 2019

Accepted: March 28, 2020

Published: March 31, 2020

Copyright (๑) 2020 by author(s) and Scientific Research Publishing Inc. This work is licensed under the Creative Commons Attribution International License (CC BY 4.0).

http://creativecommons.org/licenses/by/4.0/ (c) (i) Open Access

\begin{abstract}
Fiber optic displacement sensors are widely used in industry. Retro reflective fiber optic displacement sensor consists of parallel fibers with a reflector at a distance. Light is launched into the transmitting fiber which gets reflected by reflector. This reflected light is collected by the receiving fiber. The received light is function of the displacement of the reflector from the fiber end faces. This paper is targeted to obtain a robust design for the fiber optic displacement sensor (FODS) using well known Taguchi method. The design takes care of all noise parameters within constraints of manufacturing tolerances. The statistical data analysis is performed on the simulated results. The larger the better signal to noise quality characteristics is used to find the effect of control parameters in the data analysis. Taguchi analysis suggests dominant parameters, which affects the sensitivity of the FODS and causes immunity to noise. A source fiber inclination angle is chosen as an adjustment parameter. Other control parameters are used for fine tuning of the FODS design for achieving three qualities viz. best robustness, optimized sensitivity and robustness and best sensitivity.
\end{abstract}

\section{Keywords}

Retroreflective Fiber Optic Sensor, Taguchi Method, Optimization of Sensor Geometry

\section{Introduction}

Fiber optic sensors are used to measure different physical and chemical parameters such as pressure, temperature, strain etc. in a variety of industrial environments including oil wells, jet engines, on line and off line measurement of in 
flight parameters, power transformers, civil structures [1]. These sensors have number of unique advantages such as immunity to electromagnetic interference, small size, high temperature capability, ability to isolate the sensor head from the electronic components by very large distances and ability to multiplex number of sensors along single fiber cable [2]. In present work intensity modulated retro reflective parallel two-fiber sensor probe is developed for measuring the displacement. The sensor consists of light source (LED), photo-detector, source fiber and receiving fiber with a reflector at a distance. This sensor is well studied and number of geometries of this sensor is reported [3]. The sensor performance and accuracy of measurement strongly depend on the geometrical parameters of the probe [4]. Optimization of the sensor geometry uses series of experiments (simulations or physical) to find the parameter settings for the design that yield the predicated performance. The design is said to be robust if it is insensitive to the effects of noise variations. Taguchi techniques are most known and recurrently used for quality product design. This technique is used for robust parameter design of retro reflective fiber optic displacement sensor (FODS).

\section{Sensor Geometry and Operating Principle}

Fiber optic displacement sensor (FODS) consists of single pair parallel fibers with a reflector placed at a distance $\mathrm{Z}$ as shown in Figure 1. Light is launched into source fiber (TF) from LED which is carried up to the reflector in the form of cone. This cone of light is reflected from the reflector which is placed at a distance Z. Part of this reflected light enters the receiving fiber (RF). Amount of reflected light entering the receiving fiber ( $R F)$ depends on the distance $Z$ between the fibers and reflector. Cross section of this reflected cone of light is circular which overlaps the core of receiving fiber. The area of overlap depends upon the distance $\mathrm{Z}$. For smaller values of $\mathrm{Z}$ overlap area is almost zero causing zero received intensity. This region is called as blind region.

As distance $\mathrm{Z}$ increases, area of overlap increases causing partial overlap. The region over which this continues is called linear region. This region is called as non linear region. Blind region, linear region and non linear region are shown in Figure 2.

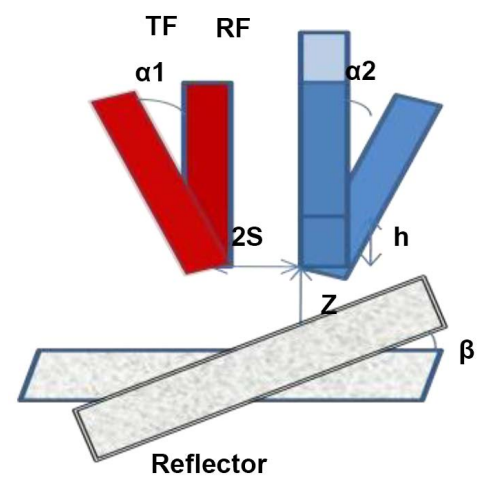

Figure 1. Control and noise factors. 


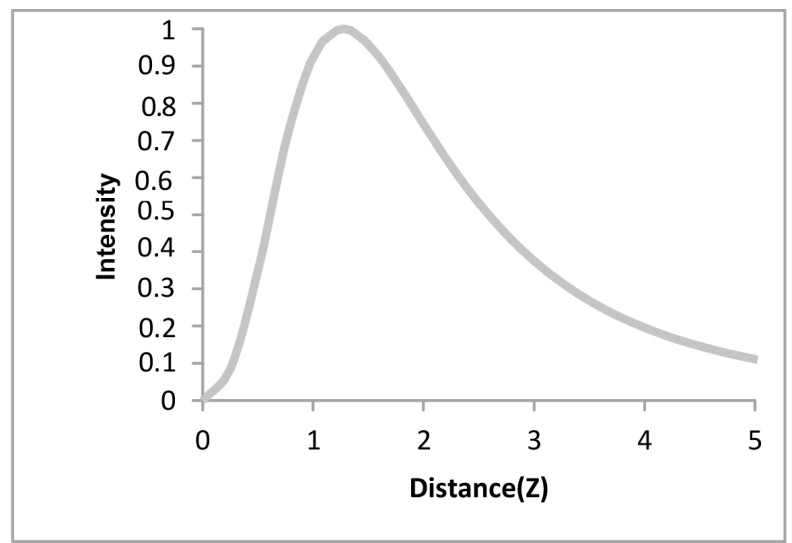

Figure 2. Sensor response.

\section{Influence of Manufacturing Tolerances on the Performance}

Performance of FODS depends on the geometrical and fabrication parameters of FODS [5]. While fabricating the FODS for a given application it is necessary to consider the manufacturing tolerances. These are related to the geometry of the FODS as shown in Figure 1. This affects the target value of the desired performance parameter of FODS for instance sensitivity in case of FODS. In addition to this there are certain parameters which are not directly related to the manufacturing process but related to the alignments and aging effects. These are called noise parameters. Sensor performance also gets affected by these parameters. Thus one has to design a FODS which is immune to noise factor variations. Taguchi technique is used to optimize the FODS design for immunity to noise factors. This technique is useful in determining the control factors so that the sensitivity of FODS does not get affected by noise factors and its value will not diverted from the target value. The design of FODS is considered as dynamic problem. In the present study, five control factors are considered at 5 levels and two noise factors are considered at 3 levels. There values are given in Table 1. Performance of FODS is analyzed by monitoring the sensitivity of sensor to decide the robust design.

For robust design first orthogonal array of control factors is chosen which comprise inner array. At the same time noise factors are chosen to form the outer array. These arrays specify the test cases to conduct the experiments i.e. used for design of experiments (DOE). These experimental results are summarized into metric called signal to noise ratio which considers how effectively the mean value (signal) of parameter has been achieved and amount of variability that has been experienced. Thus parameter having greatest effect on the performance of the product is identified. After experiments are conducted, signal to noise ratio is calculated for each test case.

This data is then analyzed statistically by using the analysis of variation (ANOVA) techniques. A parameter having largest signal to noise ratio is identified as significant contributor for achieving the best performance characteristic 
Table 1. Control Factors and noise factors.

\begin{tabular}{|c|c|c|c|c|c|}
\hline Control Factors & Level 1 & Level 2 & Level 3 & Level 4 & Level 5 \\
\hline Horizontal offset(s) in mm & “0” & “ $0.2 ”$ & “0.4” & “0.6” & “ $0.8 ”$ \\
\hline Vertical offset(h) in mm & $“-1.0 ”$ & $“-0.5 ”$ & “0” & “0.5” & “1.0” \\
\hline Source fiber angle in degrees & “0” & “5” & “10" & “15” & “20” \\
\hline Receiver fiber angle in degrees & “0” & “5” & “10" & “15” & “20” \\
\hline Core radius in $\mathrm{mm}$ & “0.1” & “0.2” & “0.3” & “0.4” & “0.5” \\
\hline
\end{tabular}

Noise Factors: Noise level 1: Inclination of reflector in degrees: “-2"; Source intensity function: "Linear". Noise level 2: Inclination of reflector in degrees: "0"; Source intensity function: "Uniform". Noise level 3: Inclination of reflector in degrees: "2"; Source intensity function: “Gaussian”.

while others having small signal to noise ratio are considered as insignificant contributors. A comprehensive design is suggested for getting the best performance. A range of results is suggested along with the expected value taking into account the error. One has to simulate or physically conduct the experiment for the suggested design and check whether result is within the expected range.

\section{Robust Design of FODS Geometry Using 1-25 Array of Taguchi Method}

Table 2 shows the complete L-25 orthogonal array for the FODS optimization. Lower part of Table 2 shows the noise factors representing 9 combinations for each row of upper part of Table 2.

Tilt of reflector is one of the noise factors. It is considered as the noise factor because reflector must be exactly parallel to the end face of the fiber.

With the defined control factors and noise factors simulations are carried out for the 25 experiments using the developed model to find the effect on the sensitivity of fiber optic displacement sensor due to variation in control factors along with noise factors.

\section{Experimental Validation of Taguchi Method for Designing Fiber Optic Sensors}

Experimental study of different architectures of fiber optic displacement sensor is carried out by construction and fabrication of sensor prototype. Different fiber sensor probes with different geometrical parameters like offset between the transmitting and receiving fiber tips(h), angle between the transmitting and receiving fiber $(\alpha)$ and separation between transmitting and receiving fiber(s) were fabricated. These probes were mounted on the basic assembly consisting of reflector and rack and pinion arrangement and their performance was studied experimentally. Figure 3 shows sensor prototype assembly with two fiber sensor geometry.

\section{Configuration Details}

The prototype consists of identical transmitting and receiving fiber with following specifications: core radius $=0.488 \mathrm{~mm}$, cladding thickness $=0.612 \mathrm{~mm}$, 
Table 2. L-25 outer arrays of noise factors.

\begin{tabular}{cccccccccc}
\hline Noise repetition factors & 1 & 2 & 3 & 4 & 5 & 6 & 7 & 8 & 9 \\
\hline Inclination of reflector in degrees & I1 & I2 & I3 & I4 & I5 & I6 & I7 & I8 & I9 \\
Source intensity function & S1 & S2 & S3 & S4 & S5 & S6 & S7 & S8 & S9 \\
\hline
\end{tabular}

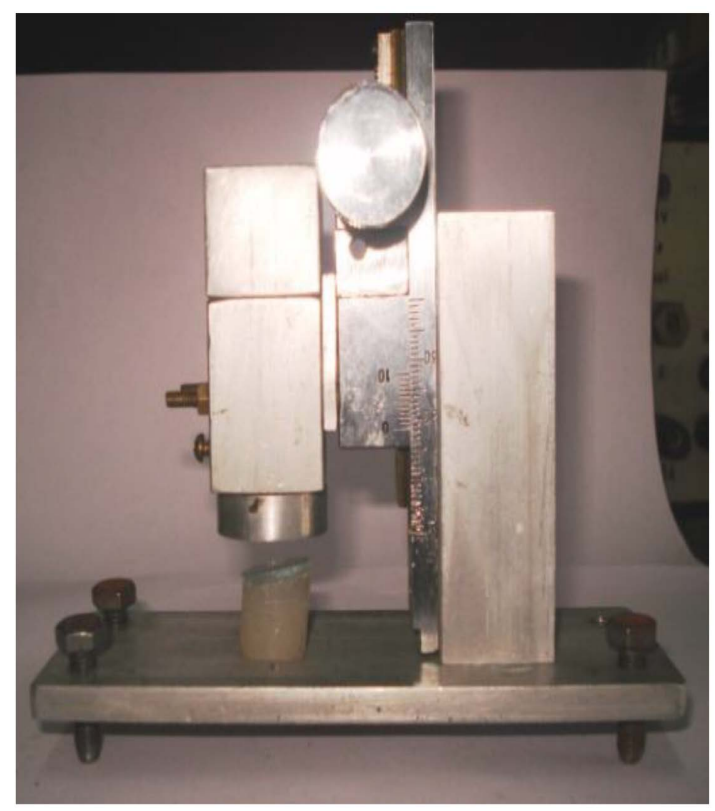

Figure 3. Photograph of prototype for fiber optic displacement sensor.

$\mathrm{NA}=0.47$, fiber diameter $=2.2 \mathrm{~mm}$, length of the fiber $=30 \mathrm{~mm}$. The fibers are enclosed in cylindrical structure fabricated in aluminum. The whole assembly can be moved with rack and pinion arrangement. Reflector is placed at a distance. Distance between the fiber end face and reflector is varied from $0 \mathrm{~mm}$ to $10 \mathrm{~mm}$ in steps of $0.2 \mathrm{~mm}$. Figure 4 shows different sensor probes fabricated with different geometrical arrangements. Details of the developed system are elaborated in further sections.

\section{Results and Discussion}

These experiments are carried out by running complete set of noise factor setting for each combination of control factor setting (at each run) using the developed mathematical model [6]. The developed mathematical model is based on the ray tracing technique. Considering the two parallel fiber geometry of the fiber optic displacement sensor, generalized co-ordinates for the source fiber and its image in the reflector is derived using the rotational transformations, reflection, vertical transformation etc. After calculating these co-ordinates, light entering the receiving fiber is calculated by successive addition of the weighted intensity of the rays entering the receiving fiber. The sensor response can be simulated for different geometrical parameters of fiber optic displacement sensor viz. 


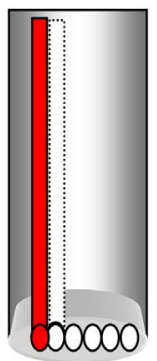

(a)

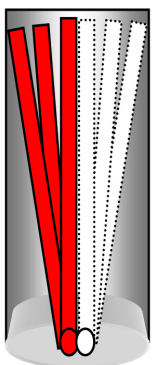

(b)

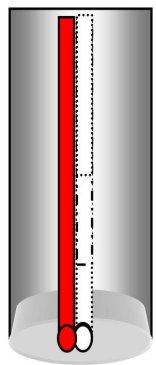

(c)

Figure 4. Schematic of sensor probe for different geometrical configurations. (a) horizontal spacing(s); (b) inclination $(\alpha)$; (c) vertical spacing(h).

vertical offset (h), horizontal offset(s), source fiber angle ( $\alpha 1)$, receiving fiber angle ( $a 2)$ and core radius(a). This model is developed for studying and analyzing sensor performance parameters for different geometrical configurations of fiber optic displacement sensor.

\subsection{Effect of Variation in Different Geometrical and Fabrication Parameters of Fiber Optic Displacement Sensors}

The effect of variation in the different geometrical and fabrication parameters of fiber optic displacement sensor on the performance of the sensor are discussed and analyzed here.

\subsection{Effect of Variation Is Horizontal Offset(s)}

There is no light entering the receiving fiber for smaller values of distance $\mathrm{Z}$ between the fiber end faces and reflector. As distance increases the reflected cone starts overlapping the receiving fiber. It shows linear variation in the amount of light entering the receiving fiber and the distance $\mathrm{Z}$. The rate of overlap of reflected cone to the receiving fiber determines the sensitivity of the sensor. The total overlap signifies the maximum intensity collected by the receiving fiber. The range of distance $\mathrm{Z}$ over which this continues is called the operating range of the sensor. Thus linear region is important as it decides sensitivity and operating range of the sensor.

Figure 5(a) shows that there is no horizontal offset between them i.e. $s=0$. The dark circles show the cross section of reflected cone at different $Z$ values from the reflector. The sensor response corresponding to this situation is explained in the previous section. Now if the receiving fiber is displaced from the source fiber for a distance $s=\mathrm{S} 1$, as shown in Figure 5(b), then the receiving fiber get overlapped by the reflected cone at larger value of $\mathrm{Z}$. This increases the blind region. The overlapping occurs towards the peripheral region of the reflected cone. The rate of overlap of the receiving fiber is smaller compared to that in $s=0$. The amount of light collected by the receiving fiber is also smaller because of the Gaussian distribution of intensity over the reflected cone. Thus 


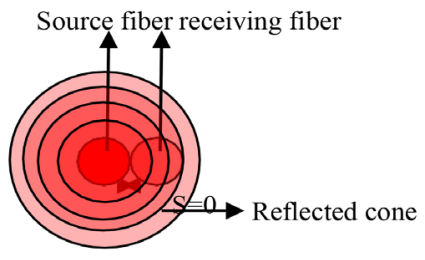

(a)

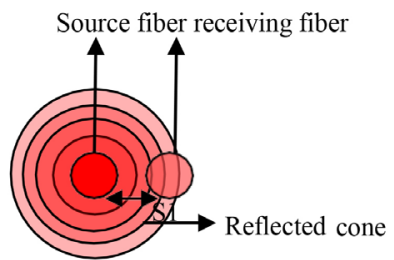

(b)

Figure 5. (a) Overlap area for $\mathrm{s}=0$; (b) Overlap area for $\mathrm{s}=\mathrm{S} 1(>0)$.

variation in the horizontal offset causes significant variation in the sensitivity of sensor.

\subsection{Effect of Source Fiber Angle ( $\alpha 1)$}

For uninclined source fiber the reflected light cone is right circular cone as shown in Figure 6(a). If the source fiber is slightly inclined then the reflected cone gets shifted slightly towards the receiving fiber as shown in the Figure 6(b). This increases the rate of overlap of receiving fiber cone with distance $Z$. This in turn increases the sensitivity of the sensor.

\subsection{Effect of Variation in Core Radius}

Increase in the core radius of source fiber and receiving fiber causes increase in the amount of light collected by the receiving fiber. Thus for small change in the distance $\mathrm{Z}$ between the fiber end face and reflector causes large change in the amount of light collected by the receiving fiber. This means that the rate of overlap of receiving fiber with reflected cone increases. This is obvious because of the increase in the core radius of the source as well as receiving fiber.

\subsection{Effect of Variation in $\mathrm{h}$}

The variation in vertical offset $h$ between the source and receiving fibers does not show any significant change in the sensitivity of sensor. The relative vertical displacement of the two fibers does not cause any variation in the amount of light intensity received by the receiving fibers. The occurrence of the maximum received intensity takes place at smaller values of $Z$ for negative offset values(receiving fiber shifted towards reflector) and for larger $\mathrm{Z}$ values for positive offset values(receiving fiber shifted away from reflector).

\subsection{Effect of Variation in Receiving Fiber Angle ( $\alpha 2)$}

Figure 7(a) and Figure 7(b) show sensor geometries with straight and inclined receiving fibers respectively. The inclination of receiving fiber causes shifting of receiving fiber core centre away from the source fiber. This reduces the overlap area of the receiving fiber core with the reflected cone. The effect of variation in receiving fiber angle does not shown any variation in the sensitivity. Simulations are carried out for the given set of 25 experiments and the sensitivity data for FODS from each run simulation of the noise factors in the outer array are 


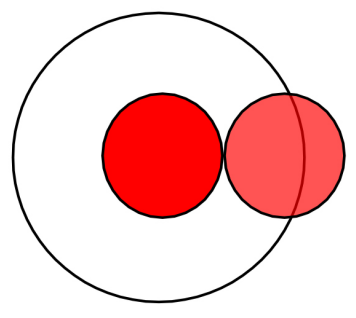

(a)

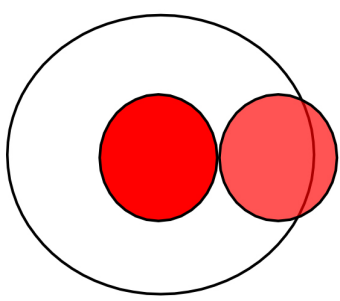

(b)

Figure 6. (a) Cross section for source angle $\alpha 1=0$; (b) Cross section for $\alpha 1>0$.

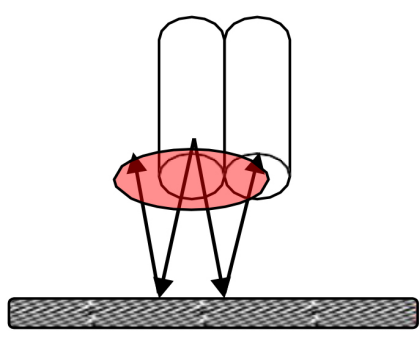

(a)

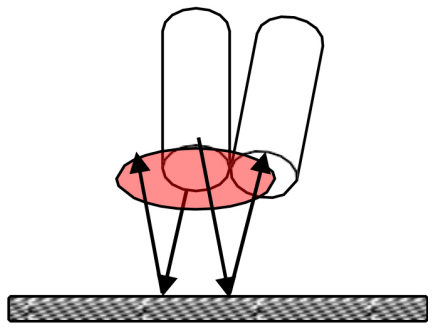

(b)

Figure 7. (a) Sensor geometry for $(\alpha 2=0)$; (b) Sensor geometry for $(\alpha 2>0)$.

usually aligned in a row, next to the factors settings for that run of the control factors in the inner array.

It is the objective of the design activity to determine the best levels of these factors to achieve the best sensitivity for FODS. Signal to noise ratio is considered as a response of the experiment, which is a measure of variation when uncontrolled noise factors are present in the system. The maximum signal to noise ratio is advocated in the Taguchi approach to maximize the performance of a system using control factors by minimizing the effect of noise variations using adjustable factors. From the ANOVA analysis it is predicted that horizontal offset(s), source fiber angle ( $\alpha 1)$ and core radius (a) are dominant parameters while vertical offset $(\mathrm{h})$, receiving fiber angle $(\alpha 2)$ are neutral or insignificant parameters. One has to find the adjustment factor so as to tune the mean to the target value. For different values of " $s$ ", it is observed that there is improvement in the linear operating range and not the sensitivity of the sensor. For different values of " $h$ " there is no effect on the sensitivity but slight increase in the linear operating range is observed. Increase in the core radius of fiber increases the linear operating range and not sensitivity. Increase in the source fiber angle improves the sensitivity of the fiber optic sensor. This is because as inclination increases the amount of light entering the receiving fiber after reflection increases w.r.t. distance i.e. rate of overlap increases. Hence source fiber angle is adjustment parameter for getting target value. Two noise factors viz. change in the source intensity function and reflector angle are considered. Effect of variation in the source intensity function decides the weight factor for the intensity which 
enters the receiving fiber after reflection. This can be adjusted by varying the source fiber angle by proper angle. Effect of variation in the reflector angle also shows change in the sensitivity of FODS. Variation in reflector angle is similar to inclining both the fibers asymmetrically due to which reflected cone becomes elliptical and the rate of overlap of reflected cone with the receiving fiber core increases which in turn increases the sensitivity of FODS. So its effect can be removed or nullified by adjusting source fiber angle.

\subsection{Fine Tuning}

In order to adjust the sensitivity exactly to the target value two other control factors viz. vertical offset ( $h$ ) and core radius (a) are considered. The adjustment is done by taking the two values for " $h$ " viz " 0 " and " 1 " and three values for "a" viz $0.1,0.3$ and 0.5 . Six experiments are arranged considering the variations in these values as shown in Table 4. After the analysis of the graphs for Nominal-the-best and Mean (Larger-the-better) different sets of control factors are found to be useful as shown in Table 3.

\subsection{Expt No 1}

The configuration is $\mathrm{s}=0, \mathrm{~h}=1.0, \alpha \mathrm{l}=20, \alpha 2=0, \mathrm{a}=0.1 \mathrm{~mm}$. It has two fibers with no horizontal offset while receiving fiber is vertically displaced by $1.0 \mathrm{~mm}$. The source fiber is inclined at an angle of 20 degree with straight receiving fiber. The radius of both the fibers is chosen as a $=0.1 \mathrm{~mm}$. As the fiber is displaced vertically, the effective distance between the reflector and the receiving fiber is $\mathrm{Z}$ $+h$. The radius of the receiving fiber is small and hence variation in the source light intensity function and reflector angle does not show much variation in the sensitivity of the sensor. Because the radius is small and fiber is displaced vertically, the rate of overlap of receiving fiber by tilted reflected cone does not show significant variation. Hence it is robust configuration. It is insensitive to variations in the noise parameters viz. reflector angle and source intensity distribution. The configuration has source fiber inclined at an angle of 20 degree. The small variation in reflector angle by 4 degree shows $1.5 \%$ variation in sensitivity for a particular type of source intensity distribution. The variation in the source intensity distribution function causes $12.5 \%$ variation in the sensitivity of the sensor.

Table 3. Predictions for the best configurations.

\begin{tabular}{|c|c|c|c|c|c|c|}
\hline Exp t no & “s" & "h" & “ $\alpha 1 "$ & “ $\alpha 2 "$ & “a” & Purpose \\
\hline 1 & “0” & “1” & “20” & “0” & “ $0.1 ”$ & Best robustness to I and S \\
\hline 2. & “0” & “0” & “20” & “0” & “0.3” & $\begin{array}{l}\text { Optimum FODS Sensitivity and } \\
\text { robustness to I and S }\end{array}$ \\
\hline 3. & “0” & “0” & “20” & “0” & “0.5” & Best FODS sensitivity \\
\hline
\end{tabular}




\subsection{Expt 2}

In this configuration, core radius is $0.3 \mathrm{~mm}$ for transmitting and receiving fibers. Thus significant variations in the intensity are observed with better sensitivity values compared to expt. 1. As the diameter of the core of the fiber is more, the intensity distribution profile of the reflected cone is spread over the core of receiving fiber. The core of transmitting fiber is larger and hence the reflected cone of light has larger diameter compared to that in expt. 1. The rate of overlap reflected cone and receiving fiber is larger thus sensitivity is more. The change in the reflector angle causes the reflected cone focused at different positions on the receiving fiber core which in turn changes the overlap rate of reflected cone and the core of receiving fiber. Thus $5 \%$ variations is in sensitivity is observed for 4 degree variations in the reflector angle while $40 \%$ variations in the sensitivity is observed for variation in the source intensity function.

\subsection{Expt 3}

In this configuration, the core radius of both fibers is $0.5 \mathrm{~mm}$. The reflected cone has larger diameter. Thus for small change in the distance $\mathrm{Z}$, receiving fiber get covered by large amount. This increases the sensitivity of the sensor. But because of the larger core diameter, source intensity distribution causes significant variation in the sensitivity of the sensor. The reflector angle variations also cause significant variations in the sensitivity. These are found to be $7.6 \%$ for source profile variations and $54 \%$ for reflector angle variation by 4 degree.

\subsection{Experimental Results and Discussion}

\section{a) Parallel fiber configuration}

Experiment is carried out for parallel fiber configuration with different horizontal offset (s) and vertical offset (h) and readings are noted for distance variation of $0 \mathrm{~mm}$ to $10 \mathrm{~mm}$. The response is analyzed to estimate the blind region, sensitivity and linear operating range at $1 \%, 5 \%$ and $10 \%$ non linearity. The results obtained are shown in Table 4.

It is observed that with separation between the fibers sensitivity decreases and range increases. This study indicates that for better sensitivity there should be minimum spacing between transmitter and receiver fiber. A suitable compromise on required range as well as the sensitivity must be made because with increased sensitivity the range is observed to be decreasing with variations in " $s$ ". Since with " $h$ " the sensitivity and range remain constant, however the blind region depends on the offset $(h)$. For large blind region large negative " $h$ " can be incorporated in the sensor design.

\section{b) Symmetrically inclined fiber configuration}

The developed sensor prototype has a facility to incline the transmitting and receiving fibers as well as reflector. Effect of inclination of fibers on the sensor performance is studied and analyzed by performing experiments. The fibers with identical parameters are inclined symmetrically at $10^{\circ}, 20^{\circ}$ and $30^{\circ}$. Similar 
Table 4. Performance parameters of different architectures of parallel fiber sensor geometry.

\begin{tabular}{cccccc}
\hline $\begin{array}{c}\text { Parallel fiber } \\
\text { Configuration }\end{array}$ & Blind region in mm & Sensitivity $(\mathrm{V} / \mathrm{mm})$ & \multicolumn{3}{c}{ Linear operating range in $\mathrm{mm}$} \\
\hline $\mathrm{s}=0.0$ & For $\mathrm{h}=\mathbf{0 . 0}$ & & $1 \%$ & $5 \%$ & $10 \%$ \\
$\mathrm{~s}=0.2$ & 2.2 & 0.332 & 0.4 & 1.6 & 1.6 \\
$\mathrm{~s}=0.4$ & 2.4 & 0.111 & 0.8 & 2.0 & 2.0 \\
$\mathrm{~s}=0.6$ & 3.6 & 0.059 & 0.8 & 2.4 & 2.8 \\
$\mathrm{~s}=0.8$ & 4.0 & 0.034 & 1.2 & 3.2 & 3.2 \\
$\mathrm{~s}=1.0$ & 4.4 & 0.021 & 1.6 & 3.2 & 3.6 \\
\hline $\mathrm{h}=-3.0$ & 5.6 & 0.014 & 1.2 & 4.0 & 4.4 \\
$\mathrm{~h}=-2.0$ & For $\mathrm{s}=0.0$ & & & & \\
$\mathrm{~h}=-1.0$ & 5.2 & 0.321 & 0.4 & 1.2 & 1.2 \\
$\mathrm{~h}=0.0$ & 4.0 & 0.299 & 0.4 & 1.2 & 1.6 \\
$\mathrm{~h}=+1.0$ & 3.6 & 0.321 & 0.4 & 1.2 & 1.8 \\
$\mathrm{~h}=+2.0$ & 2.2 & 0.333 & 0.4 & 1.2 & 2.0 \\
$\mathrm{~h}=+3.0$ & 1.8 & 0.322 & 0.4 & 1.2 & 2.1 \\
\hline & 1.5 & 0.325 & 0.4 & 1.2 & 2.1 \\
\hline
\end{tabular}

experiments are carried out for reflector tilt from $-10^{\circ}$ to $+10^{\circ}$. The performance parameters are tabulated as shown in Table 5 for the sensor geometry with symmetrically inclined fibers as well as tilted reflector.

Larger sensitivity can be obtained with higher inclination angle between the fiber and high reflector tilt. These effects together with the required specifications would then pave the way for optimized.

\section{c) Asymmetrically inclined fiber configuration}

With an idea of improvement in sensitivity in mind, we proceed further by inclining the fibers asymmetrically i.e. transmitting fiber with angle $\alpha 1$ and receiving fiber $\alpha 2$. Experiments are carried out for different values of $\alpha 1$ and $\alpha 2$. The sensor responses are analyzed for dependence of blind region, sensitivity and linear operating range of $1 \%, 5 \%$ and $10 \%$ non linearity. Table 6 shows the effect of asymmetric inclination of fibers on performance parameters.

Keeping $\alpha 1=0^{\circ}$, if receiving fiber angle $\alpha 2$ is increased from $2^{\circ}$ to $10^{\circ}$, blind region decreases. Sensitivity of the sensor decreases by $22 \%$ and linear operating range increases by $20 \%$. However, if $\alpha 2=0^{\circ}$, the inclination of transmitting fiber angle $\alpha 1$ from $2^{\circ}$ to $10^{\circ}$, blind region decreases. However, 26\% improvement in sensitivity of the sensor is observed and linear operating range decreases by $20 \%$.

\section{Conclusion}

Two-fiber retro reflective fiber optic displacement sensor is analyzed for optimized and robust design for best sensitivity. Taguchi method is used for the 
Table 5. Performance parameters with symmetrically inclined fibers and incined reflector sensor geometry.

\begin{tabular}{cccccc}
\hline $\begin{array}{c}\text { Inclined fiber } \\
\text { Configuration }\end{array}$ & Blind region in mm & Sensitivity $(\mathrm{V} / \mathrm{mm})$ & \multicolumn{3}{c}{ Linear operating range in mm } \\
\hline \multicolumn{2}{c}{ With Symmetrically inclined fibers } & 0.333 & $1 \%$ & $5 \%$ & $10 \%$ \\
\hline$\alpha=0^{\circ}$ & 2.2 & 0.449 & 0.4 & 1.2 & 1.2 \\
$\alpha=5^{\circ}$ & 1.6 & 0.898 & 0.4 & 0.8 & 1.0 \\
$\alpha=10^{\circ}$ & 1.4 & 1.88 & 0.4 & 0.6 & 0.8 \\
$\alpha=15^{\circ}$ & 0.8 & 3.98 & 0.4 & 0.5 & 0.8 \\
$\alpha=20^{\circ}$ & 0.6 & 6.66 & 0.2 & 0.4 & 0.8 \\
$\alpha=25^{\circ}$ & 0.0 & 7.09 & 0.2 & 0.4 & 0.8 \\
$\alpha=30^{\circ}$ & 0.0 & & & & \\
\hline$\beta=-2^{\circ}$ & With inclined reflector & 0.122 & 1.2 & 1.4 & 1.8 \\
$\beta=-1^{\circ}$ & 1.4 & 0.225 & 0.8 & 1.4 & 1.6 \\
$\beta=0^{\circ}$ & 1.6 & 0.388 & 0.4 & 1.2 & 1.4 \\
$\beta=1^{\circ}$ & 2.2 & 0.556 & 0.4 & 1.0 & 1.2 \\
$\beta=2^{\circ}$ & 1.4 & 0.876 & 0.4 & 1.0 & 1.2 \\
\hline & 1.6 & & & & \\
\hline
\end{tabular}

Table 6. Performance parameters with asymmetrically inclined fibers sensor geometry.

\begin{tabular}{ccccccc}
\hline $\begin{array}{l}\text { Inclined fiber } \\
\text { Configuration }\end{array}$ & \multicolumn{2}{c}{ Blind region in $\mathrm{mm}$} & $\begin{array}{c}\text { Sensitivity } \\
(\mathrm{V} / \mathrm{mm})\end{array}$ & \multicolumn{3}{c}{ Linear operating range in $\mathrm{mm}$} \\
\hline \multicolumn{2}{c}{ With Asymmetrically inclined fibers } & $1 \%$ & $5 \%$ & $10 \%$ \\
\hline$\alpha 1$ & $\alpha 2$ & & & & \\
\hline $0^{\circ}$ & $2^{\circ}$ & 1.0 & 1.07 & 0.4 & 1.2 & 1.6 \\
$0^{\circ}$ & $5^{\circ}$ & 0.4 & 0.766 & 0.4 & 1.4 & 1.8 \\
$0^{\circ}$ & $10^{\circ}$ & 0.0 & 0.221 & 0.4 & 1.4 & 2.0 \\
$2^{\circ}$ & $0^{\circ}$ & 4.0 & 0.115 & 0.4 & 1.6 & 2.0 \\
$5^{\circ}$ & $0^{\circ}$ & 2.2 & 0.131 & 0.2 & 1.6 & 1.8 \\
$10^{\circ}$ & $0^{\circ}$ & 1.8 & 0.145 & 0.2 & 0.8 & 1.6 \\
\hline
\end{tabular}

analysis. Nominal-the-best and Mean (larger-the-better) is used for finding the best robust, optimized sensitivity and best sensitivity configuration of the fiber optic displacement sensor. Experimental results are also in good agreement with simulation experiment done using Taguchi method.

\section{Acknowledgements}

One of the authors (SSP) wishes to thank HOD, Department of Electronic Science, University of Pune for allowing me to make use of the laboratory. SSP is also thankful to Principal A. G. College, Pune and thankful to UGC as well. 


\section{Conflicts of Interest}

The authors declare no conflicts of interest regarding the publication of this paper.

\section{References}

[1] Udd, E. (1995) An Overview of Fiber Optic Sensors. Review of Scientific Instruments, 66, 4015-4030. https://doi.org/10.1063/1.1145411

[2] Culshaw, B. and Dakin, J. (Eds) (1989) Optical Fiber Sensor Systems and Applications. Vol. 2, Artech House, Norwood.

[3] Faria, J.B. (1998) A Theoretical Analysis of the Bifurcated Fiber Bundle Displacement Sensor. IEEE Transactions on Instrumentation and Measurement, 47, 742-747. https://doi.org/10.1109/19.744340

[4] Buchade, P.B. and Shaligram, A.D. (2006) Experimental and Simulation Studies of Inclined Two Fiber Displacement Sensor. Sensors and Actuators, A28, 312-316. https://doi.org/10.1016/j.sna.2006.02.002

[5] Buchade, P.B. and Shaligram, A.D. (2007) Influence of Fiber Geometry on the Performance of Two-Fiber Displacement Sensor. Sensors and Actuators, A136, 199-204. https://doi.org/10.1016/j.sna.2006.11.020

[6] Patil, S.S. and Shaligram, A.D. (2011) Modeling and Experimental Studies on Retro-Reflective Fiber Optic Micro-Displacement Sensor with Variable Geometrical Properties. Sensors and Actuators, A172, 428-433.

https://doi.org/10.1016/j.sna.2011.10.006 